\title{
Wirtschaftliche Praxisführung: Diese Kosten sind im EBM gesondert berechnungsfähig
}

_ Die Allgemeinen Bestimmungen des EBM-Kapitels I legen unter Punkt 7.3 diverse Kosten fest, die in den Gebührenordnungspositionen nicht enthalten sind, soweit es dortselbst nichts anderes bestimmt ist. Dazu gehören Kosten für Arzneimittel, Verbandmittel, Materialien, Instrumente, Gegenstände und Stoffe, die nach der Anwendung verbraucht sind oder die der Patient zur weiteren Verwendung behält, ferner Kosten für Infusionsbestecke, -katheter, -nadeln und Biopsienadeln zur einmaligen Verwendung. Auch Telefonate des behandelnden Arztes mit einem Krankenhaus können extra berechnet werden, wenn es um eine erforderliche stationäre Behandlung geht.

\section{MMW-KOMMENTAR}

Wegen der Geringfügigkeit dürfte insbesondere der letzte Punkt, der die Möglichkeit eröffnet, Telefonkosten im Zusammenhang mit einem Krankenhausaufenthalt des Patienten zu berechnen, häufig in Vergessenheit geraten. Trotzdem gilt es, hier nichts zu verschenken - denn alle Kosten, die geltend gemacht werden können, werden auch wie eingefordert und unbudgetiert vergütet.

Das Problem ist die Unübersichtlichkeit bei den Regelungen, die sich aus dem Passus "soweit nichts anderes bestimmt ist" ergibt. Der Punkt 7.1 der Allgemeinen Bestimmungen ist ziemlich genau, wenn es um die Posten geht, die in den Gebührenordnungspositionen bereits enthalten sind. Das sind neben allgemeinen Praxiskosten wie Miete und Reinigung und den Betriebskosten ärztlicher Instrumente und Apparaturen auch die Kosten für die meisten Einmalprodukte, nämlich Spritzen, Kanülen, Trachealtuben, Absaugkatheter, Handschuhe, Rasierer, Harnblasenkatheter, Skalpelle, Proktoskope, Darmrohre,

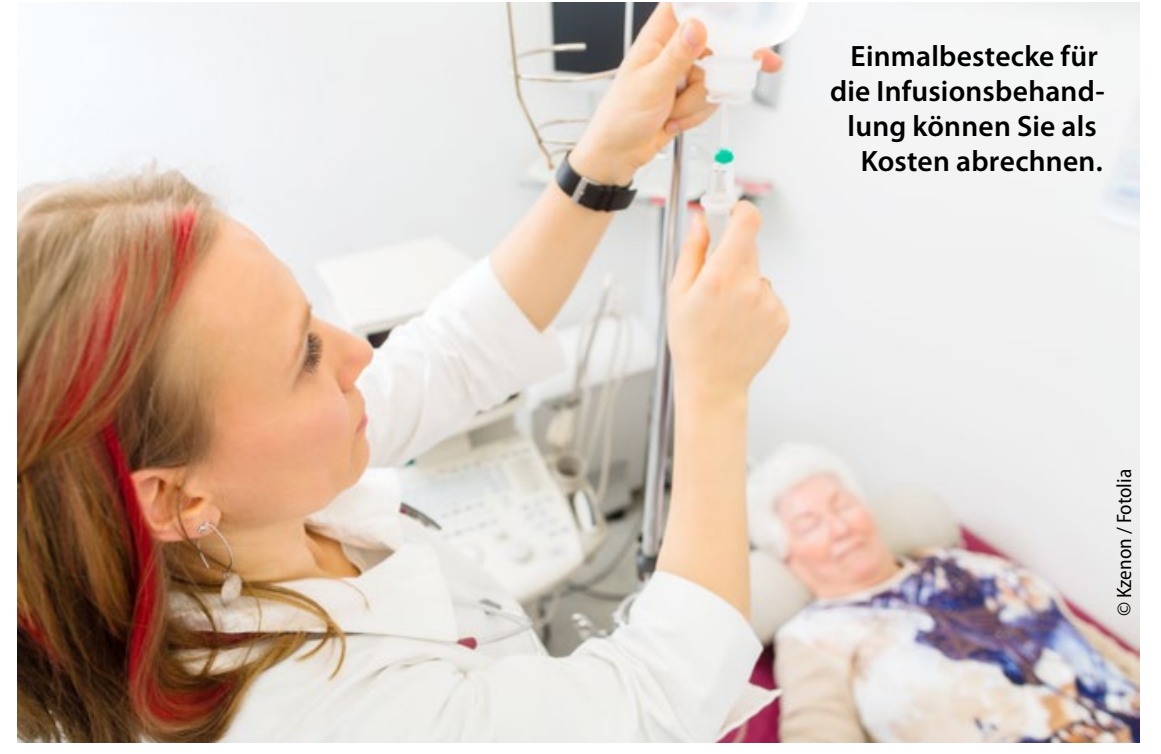

Spekula, Küretten und Abdecksets. Auch die Kosten fürs Labor und für Fotoaufnahmen trägt die Praxis selbst. Das gilt sogar für Versand- und Transportkosten - mit einigen Ausnahmen, nämlich Arztbriefe und Post im Zusammenhang mit Langzeit-EKG, Laboruntersuchungen, Zytologie, Histologie, Zytound Molekulargenetik, Strahlendiagnostik und -therapie sowie der Anwendung radioaktiver Substanzen.

Daneben gibt es den Sprechstundenbedarf, der auf Landesebene vertraglich geregelt wird-leider nicht bundesweit einheitlich. Von der KV gibt es eine Liste der anforderungsfähigen Artikel. Beispiel Einmalinstrumente: Sie können berechnungsfähig sein, wenn man sie als Sprechstundenbedarf bezieht. Dafür muss allerdings auf Bundesebene im EBM oder in den regionalen Gesamtverträgen eine eindeutige Regelung existieren. In allen anderen Fällen, in denen man auf mehrfach verwendbare Instrumente zurückgreifen könnte, besteht dann grundsätz- lich kein Erstattungsanspruch. Das gilt nicht nur gegenüber der KV oder der Krankenkasse, sondern auch gegenüber dem Patientenweil die Instrumente ja bereits mit der abgerechneten Gebührenordnungsposition abgegolten sind. Es wäre also eine unzulässige Doppelvergütung. Man muss daher bei jeder Leistung darauf achten, ob ggf. aus der Legende hervorgeht, dass die Kosten mit der Erbringung abgegolten sind.

Eine Ausnahme machen hier Einmalinfusionsbestecke, -katheter und-nadeln (also z. B. "Butterfly" oder "Braunüle"), die in den Allgemeinen Bestimmungen erwähnt sind und demnach gesondert berechnungsfähig sind oder über den Sprechstundenbedarf bezogen werden können.

Interessanterweise gibt es eine identische Regelung in der GOÄ. Bei der Infusionsbehandlung eines Privatpatienten sollte man deshalb nicht vergessen, diese Kosten, ggf. zusammen mit den Arzneimittelkosten, in die Rechnung aufzunehmen. 